\title{
Detailed abundances in a sample of very metal-poor stars ${ }^{\star}$
}

\author{
P. François ${ }^{1,2}$, S. Wanajo ${ }^{3,4,5}$, E. Caffau ${ }^{6}$, N. Prantzos ${ }^{7}$, W. Aoki ${ }^{9}$, M. Aoki ${ }^{8}$, P. Bonifacio ${ }^{6}$, M. Spite ${ }^{6}$, and F. Spite ${ }^{6}$ \\ ${ }^{1}$ GEPI, Observatoire de Paris, PSL Research University, CNRS, 61 Avenue de l'Observatoire, 75014 Paris, France \\ e-mail: patrick.francois@obspm.fr \\ ${ }^{2}$ UPJV, Université de Picardie Jules Verne, 33 rue St Leu, 80080 Amiens, France \\ ${ }^{3}$ Max Planck Institute for Gravitational Physics (Albert Einstein Institute), Am Mühlenberg 1, Potsdam-Golm 14476, Germany \\ ${ }^{4}$ iTHEMS Research Group, RIKEN, Wako, Saitama 351-0198, Japan \\ 5 Department of Engineering and Applied Sciences, Faculty of Science and Technology, Sophia University, 7-1 Kioicho, Chiyoda-ku, \\ Tokyo 102-8554, Japan \\ ${ }^{6}$ GEPI, Observatoire de Paris, PSL Research University, CNRS, Place Jules Janssen, 92190 Meudon, France \\ ${ }^{7}$ Institut d'Astrophysique de Paris, UMR7095 CNRS, Univ. P. \& M. Curie, 98bis Bd. Arago, 75104 Paris, France \\ ${ }^{8}$ European Southern Observatory, Karl-Schwarzschild-Str. 285748 Garching bei Muenchen, Germany \\ ${ }^{9}$ National Observatory of Japan, Mitaka, Tokyo, Japan
}

Received 26 March 2020 / Accepted 8 July 2020

\begin{abstract}
Context. Unevolved metal-poor stars bore witness to the early evolution of the Galaxy, and the determination of their detailed chemical composition is an important tool to understand its chemical history. The study of their chemical composition can also be used to constrain the nucleosynthesis of the first generation of supernovae that enriched the interstellar medium.

Aims. We aim to observe a sample of extremely metal-poor star (EMP stars) candidates selected from the Sloan Digital Sky Survey data release 12 (SDSS DR12) and determine their chemical composition.

Methods. We obtained high-resolution spectra of a sample of five stars using HDS on Subaru telescope and used standard 1D models to compute the abundances. The stars we analysed have a metallicity $[\mathrm{Fe} / \mathrm{H}]$ of between -3.50 and -4.25 dex.

Results. We confirm that the five metal-poor candidates selected from low-resolution spectra are very metal poor. We present the discovery of a new ultra metal-poor star (UMP star) with a metallicity of $[\mathrm{Fe} / \mathrm{H}]=-4.25$ dex (SDSS J1050032.34-241009.7). We measured in this star an upper limit of lithium $(\log (\mathrm{Li} / \mathrm{H}) \leq 2.0$. We found that the four most metal-poor stars of our sample have a lower lithium abundance than the Spite plateau lithium value. We obtain upper limits for carbon in the sample of stars. None of them belong to the high carbon band. We measured abundances of $\mathrm{Mg}$ and $\mathrm{Ca}$ in most of the stars and found three new $\alpha$-poor stars.
\end{abstract}

Key words. stars: Population II - Galaxy: abundances - Galaxy: halo

\section{Introduction}

Metal-poor stars bore witnesses to the early evolution of our Galaxy and provide important clues as to the formation of the first objects in the Universe. Detailed abundance analyses of their atmosphere reveal observational details that can be compared to theoretical models for nucleosynthesis in the first metal-poor massive stars exploding as supernovae. The trends of their abundance ratios as a function of metallicity can be used to trace the chemical history of our Galaxy. A thorough discussion of the main scientific goals of the study of metal-poor stars can be found in several review articles (see e.g. Beers \& Christlieb 2005; Frebel \& Norris 2015).

First misidentified as early-type stars (e.g. Adams et al. 1935), the metal-poor stars revealed their true nature as old metal-poor stars only through high-resolution spectroscopic analysis (Chamberlain \& Aller 1951). The catalogue of highvelocity stars of Roman(1955) was extensively used as a source of low-metallicity stars (e.g. Greenstein et al. 1957; Wallerstein \& Helfer 1959; Wallerstein 1962; Wallerstein et al. 1963). Although this field of research was largely dominated by the

\footnotetext{
^ Based on data collected at Subaru Telescope, which is operated by the National Astronomical Observatory of Japan.
}

work of astronomers in the United States, a growing interest also began in Europe (e.g. Baschek 1963; Cayrel \& Fringant 1964).

The search for the most metal-poor stars saw great progress with the survey work of Beers et al. (1985) and the discovery of several very metal-poor stars with $[\mathrm{Fe} / \mathrm{H}] \leq-3.00 \mathrm{dex}$ (Norris et al. 1993). The survey of these latter authors made use of a temperature index based on the $\mathrm{H} \beta$ hydrogen absorption line and a metallicity index from the strong $\mathrm{Ca} \mathrm{H} \& \mathrm{~K}$ lines which can be detected and measured from low-resolution spectra even at low metallicity. High-resolution follow-up observations of metal-poor candidates led to a large number of publications. Among them were the series of articles from the First Stars ESO Large programme (Prog ID 165.N-0276) published by the Cayrel's group (Bonifacio et al. 2009; Cayrel et al. 2004, and references therein). These latter authors also revealed the rarity of the very metal-poor stars as shown by the metallicity distribution function, implying that a larger and deeper survey was needed to find new very metal-poor candidates. The Hamburg ESO survey, whose first aim was the search for new quasars, has also been very successful in the search and discovery of several stars with $[\mathrm{Fe} / \mathrm{H}]$ with metallicity lower than -4.00 dex (Christlieb et al. 2008, and references therein). More recently, the Sloan Digital Sky Survey (SDSS) spectroscopic survey was used to identify new metal-poor candidates (Helmi et al. 2003). Ludwig et al. (2008) developed an analysis tool that allows us to estimate 
Table 1. Observation log.

\begin{tabular}{lcccc}
\hline \hline Object & $g$ magnitude & Observation date & Exp. time $(\mathrm{s})$ & $S / N$ at $480 \mathrm{~nm}$ \\
\hline SDSS J081554.26+472947.5 & 17.06 & $2017-04-05 T 06: 03$ & 3600 & 25 \\
SDSS J081554.26+472947.5 & 17.06 & $2017-04-05 T 07: 04$ & 3600 & 30 \\
SDSS J081554.26+472947.5 & 17.06 & $2017-04-05 T 08: 05$ & 3600 & 30 \\
SDSS J091753.19+523004.9 & 18.58 & $2017-04-06 T 05: 55$ & 3600 & 15 \\
SDSS J091753.19+523004.9 & 18.58 & $2017-04-06 T 06: 56$ & 3600 & 15 \\
SDSS J091753.19+523004.9 & 18.58 & $2017-04-06 T 07: 57$ & 3600 & 15 \\
SDSS J105002.34+242109.7 & 18.04 & $2017-04-05 T 09: 10$ & 3600 & 20 \\
SDSS J105002.34+242109.7 & 18.04 & $2017-04-05 T 10: 11$ & 3600 & 20 \\
SDSS J105002.34+242109.7 & 18.04 & $2017-04-06 T 09: 05$ & 3600 & 25 \\
SDSS J105002.34+242109.7 & 18.04 & $2017-04-06 T 10: 05$ & 3600 & 20 \\
SDSS J105002.34+242109.7 & 18.04 & $2017-04-07 T 05: 46$ & 3600 & 25 \\
SDSS J105002.34+242109.7 & 18.04 & $2017-04-07 T 06: 47$ & 3335 & 25 \\
SDSS J124304.19-081230.6 & 18.25 & $2017-04-05 T 11: 22$ & 3600 & 15 \\
SDSS J124304.19-081230.6 & 18.25 & $2017-04-05 T 12: 23$ & 3600 & 10 \\
SDSS J124304.19-081230.6 & 18.25 & $2017-04-06 T 11: 11$ & 3600 & 20 \\
SDSS J124304.19-081230.6 & 18.25 & $2017-04-06 T 12: 12$ & 3600 & 15 \\
SDSS J124304.19-081230.6 & 18.25 & $2017-04-07 T 08: 00$ & 3600 & 20 \\
SDSS J124304.19-081230.6 & 18.25 & $2017-04-07 T 09: 00$ & 3600 & 20 \\
SDSS J153346.28+155701.8 & 16.91 & $2017-04-05 T 13: 29$ & 3000 & 20 \\
SDSS J153346.28+155701.8 & 16.91 & $2017-04-05 T 14: 20$ & 3000 & 20 \\
SDSS J153346.28+155701.8 & 16.91 & $2017-04-06 T 13: 16$ & 3300 & 20 \\
SDSS J153346.28+155701.8 & 16.91 & $2017-04-06 T 14: 11$ & 3300 & 25 \\
\hline
\end{tabular}

the metallicity of Turn Off (TO) stars from the low-resolution SDSS spectra. Follow-up observations on large telescopes have been used to confirm their metallicity and measure abundance ratios. This detection method has been quite successful and led to the discovery of several interesting very metal-poor stars (Caffau et al. 2011a,b, 2012, 2014, 2016; Bonifacio et al. 2015, 2018; François et al. 2018). Other surveys have also been used to detect metal-poor stars, such as the Apache Point Observatory Galactic Evolution Experiment (APOGEE, Majewski et al. 2016), a survey toward the galactic centre, where the stellar density allows large multiplex spectroscopic observations. Further examples are the Radial Velocity Experiment (RAVE) survey whose first aim is the study of the galactic dynamics from a radial-velocity census of stars (Steinmetz et al. 2006), and the Skymapper Sky Survey (Keller et al. 2007; Wolf et al. 2018) whereby wide field imaging is performed in five wide bands and a narrow band centred on $\mathrm{Ca} \mathrm{H} \& \mathrm{~K}$ absorption lines using the Skymapper telescope. The photometric SkyMapper Southern Sky Survey (Keller et al. 2007) discovered two of the most extremely iron-poor stars: SMSS J031300.36-670839.3 (Keller et al. 2014) and SMSS J160540.18-144323.1 (Nordlander et al. 2019). More recently, the PRISTINE survey (Starkenburg et al. 2017) based on Canada France Hawaii telescope (CFHT) large field imaging uses a dedicated narrow band filter centred on Ca H\&K absorption lines combined with SDSS broad-band $g$ and $i$ photometry. The common point between these different sources of metal-poor star candidates is that they require high-resolution spectroscopic follow-up observations to confirm their low metallicity and determine their detailed chemical composition.

From the recent analysis of SDSS DR12 data, we detected new extremely metal-poor candidates that have never been observed at high resolution. In this article, we report the detailed analysis of five new extremely metal-poor candidates observed with the High Dispersion Spectrograph (HDS) installed on the
Subaru telescope atop Mauna Kea volcano in Hawaii. Similar observations have been conducted for a second set of stars visible from the southern hemisphere using the X-shooter spectrograph installed on the UT2 (KUEYEN) at the ESO very large telescope (VLT) on Cerro Paranal in Chile in the framework of a french-japanese collaboration. The results have been published in François et al. (2018).

\section{Observation}

Observations presented here were carried out with HDS installed on the Subaru telescope (Noguchi et al. 2002). The wavelength coverage goes from 4084 to $6892 \AA$. A binning $2 \times 2$ has been adopted leading to a resolution of about 40000 . The logbook of the observations is given in Table 1. Standard data reduction procedures were carried out with the IRAF Echelle package ${ }^{1}$. Care was taken to remove the sky background as most of the exposures were affected by the moon illumination.

Figure 1 shows the spectra of the stars of our sample centred on the magnesium triplet. The continuum level of the four spectra located in the lower part of the plot has been shifted for clarity. The spectra are presented with decreasing metallicity from the top to the bottom of the figure.

\section{Stellar parameters}

Stellar parameters were derived taking into account the SDSS photometry. The effective temperatures in Table 2 were computed by Caffau et al. (2013). The effective temperature was derived from the photometry using the $(g-z)_{0}$ colour and the calibration described in Ludwig et al. (2008) taking into

\footnotetext{
1 IRAF is distributed by the National Optical Astronomy Observatories, which is operated by the Association of Universities for Research in Astronomy, Inc. under cooperative agreement with the National Science Foundation.
} 
Table 2. Adopted stellar parameters for the list of targets.

\begin{tabular}{lccccc}
\hline \hline Object & $T_{\text {eff }}$ & $\log g$ & {$[\mathrm{Fe} / \mathrm{H}]$} & $\xi\left(\mathrm{km} \mathrm{s}^{-1}\right)$ & $v_{r}\left(\mathrm{~km} \mathrm{~s}^{-1}\right)$ \\
\hline SDSS J081554.26+472947.5 & 6066 & 4.00 & -4.00 & 1.5 & -120 \\
SDSS J091753.19+523004.9 & 5858 & 4.00 & -3.50 & 1.5 & -37 \\
SDSS J105002.34+242109.7 & 5682 & 4.00 & -4.00 & 1.5 & -126 \\
SDSS J124304.19-081230.6 & 5488 & 4.00 & -3.50 & 1.5 & 154 \\
SDSS J153346.28+155701.8 & 6375 & 4.00 & -3.50 & 1.5 & -283 \\
\hline
\end{tabular}

Notes. The last column gives the measured radial velocity of the stars after correction from the barycentric velocity.

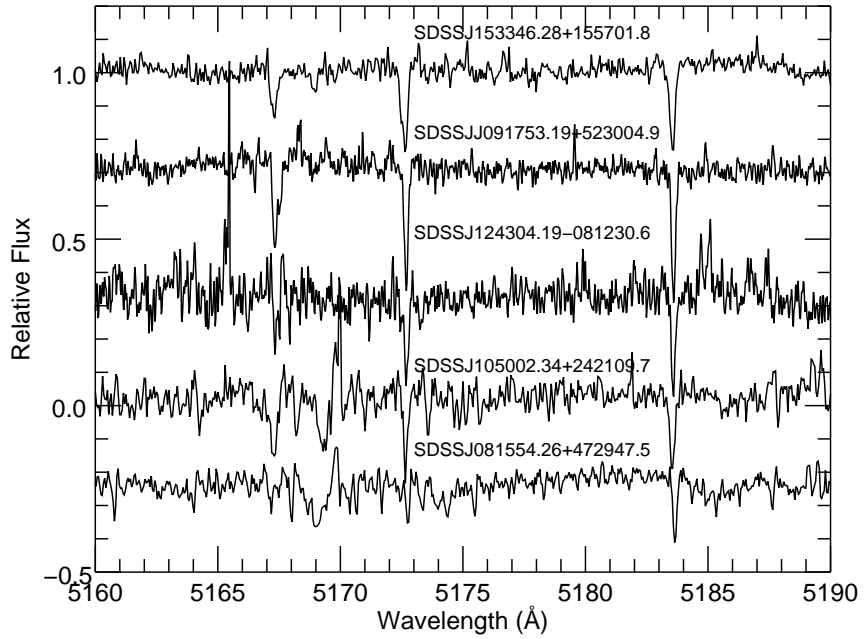

Fig. 1. Parts of the HDS Subaru spectra centred on the magnesium triplet. The continuum of the four spectra located in the lower part of the plot has been shifted downwards for clarity. The two lower spectra reveal the presence of residuals of the sky spectrum in the region 5168-5170 and 5185-5186 А.

account the reddening according to the Schlegel et al. (1998) extinction maps and corrected as in Bonifacio et al. (2000). The Gaia parallaxes (Arenou et al. 2018; Gaia Collaboration 2018) for our stars are imprecise. SDSSJ081554.26+472947.5 and SDSSJ091753.19+523004.9 parallaxes have a relative error smaller than $\sim 20 \%$. SDSSJ124304.19-081230.6 and SDSSJ153346.28+155701.8 parallaxes have a relative error of the order of $\sim 50 \%$. One star (SDSSJ105002.34+242109.7) has a negative parallax. In order to obtain insight into the luminosity of this star, we used the distance estimates of Bailer-Jones et al. (2018). Figure 2 shows $(B P-R P)_{0}$ versus the absolute $G_{0}$ magnitude. The red symbols represent the stars of our sample assuming

$E(P B-R P)=(B P-R P)-1.289445 \times E_{(B-V) \_S a n d F}$

and

$G_{0}=G-0.85926 \times A V_{\mathrm{SandF}}$,

where $E_{(B-V) \_S a n d F}$ and $A V_{\text {SandF }}$ are obtained from the maps of Schlafly \& Finkbeiner (2011). We have also plotted in Fig. 2 a PARSEC (PAdova and TRieste Stellar Evolution Code) (Bressan et al. 2012) isochrone of metallicity $-2.5 \mathrm{dex}$ and an age of 12 Gyr. The location of the stars clearly reveals that they are not giant. Indeed, the $G_{0}$ for our stars are between 3.0 and 6.5 which corresponds to $\log g$ ranging from $\simeq 3.5$ to 4.7 based on the isochrone. This confirms that four of our stars are dwarfs and

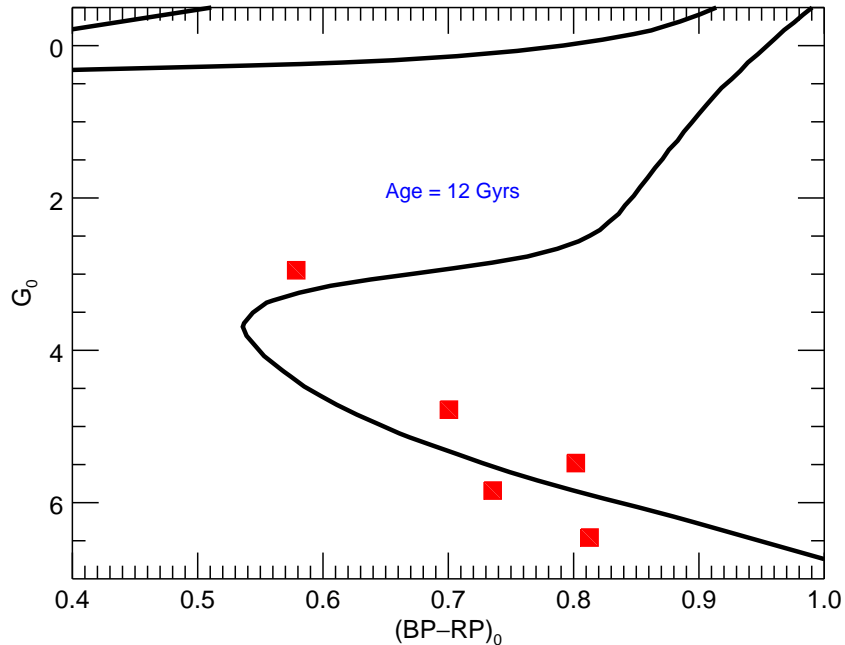

Fig. 2. Comparison of the location of the stars and a PARSEC (Bressan et al. 2012) isochrone of metallicity -2.5 and age $12 \mathrm{Gyr}$ on a $G_{0}$ magnitude versus $(B P-R P)_{0}$ magnitude diagram. The isochrone on the plot has been computed for an age of $12 \mathrm{Gyr}$ and a metallicity of -2.5 dex. Here we show BP-RP (BP and RP are the respective magnitudes measured by the two low-resolution spectrographs, the Blue Photometer (BP) and the Red Photometer (RP) onboard the Gaia satellite) versus the absolute $\mathrm{G}$ magnitude. The red symbols represent our stars.

one seems slightly evolved. Adopting $\log g=4.00$ for the gravity of our stars is suited for our analysis. We reiterate that the selection of the stars is based on the dereddened $(g-z)$ and $(u-g)$ colours: $0.18 \leq(g-z)_{0} \leq 0.70$ and $(u-g)_{0} \geq 0.70$. As discussed in Bonifacio et al. (2012), this selects the stars of the halo turn-off and excludes the majority of the white dwarf stars. A microturbulent velocity of $1.5 \mathrm{~km} \mathrm{~s}^{-1}$, which is suitable for stars with $\log$ $g=4$ dex, has been adopted following the results of Barklem et al. (2005). The metallicities shown in Table 2 were computed by Caffau et al. (2013) using the code MyGisFoS (Sbordone et al. 2010) and the SDSS spectra of the stars.

From the radial velocities given in Table 2 and using the publicly licensed code GalPot ${ }^{2}$, which is described by Dehnen \& Binney (1998), we computed the kinematic properties of our sample of stars and some of the quantities derived from their Galactic orbits. The results are shown in Table 3. In this table, $L_{z}$ is the angular momentum, $R$ is the galactocentric radius (cylindrical), $R_{\min }$ and $R_{\max }$ are the minimum and the maximum values of the galactocentric radius (cylindrical) of the orbit, respectively, and $E$ is the energy of the orbit. $Z_{\max }$ is the maximum galactocentric height of the orbit. The space velocities $(U, V, W)$ are with respect to the local standard of rest, $U$ is

2 GalPot can be found at https://github.com/ PaulMcMillan-Astro/GalPot 
Table 3. Orbit parameters.

\begin{tabular}{ccccccccc}
\hline \hline Object & $R_{\min }$ & $R_{\max }$ & $Z_{\max }$ & Energy & $L_{Z}$ & $X$ & $Y$ & $Z$ \\
\cline { 2 - 9 } SDSS J081554.26+472947.5 & 0.08 & 10.11 & 10.42 & -150171 & -19.28 & 10.06 & -0.26 & 1.26 \\
SDSS J091753.19+523004.9 & 0.49 & 14.80 & 14.99 & -142622 & 156.89 & 9.21 & -0.27 & 0.99 \\
SDSS J105002.34+242109.7 & 2.15 & 29.68 & 7.58 & -116458 & -1061.98 & 9.25 & 0.65 & 2.38 \\
SDSS J124304.19-081230.6 & 1.61 & 16.21 & 2.86 & -145572 & 740.35 & 7.69 & 0.92 & 1.50 \\
SDSS J153346.28+155701.8 & 4.31 & 11.57 & 8.23 & -149829 & 1232.96 & 5.18 & -1.40 & 4.10 \\
Object & $V_{X}$ & $V_{Y}$ & $V_{Z}$ & $R$ & $\phi$ & $V_{r}$ & $V_{Z}$ & $V_{\phi}$ \\
\hline SDSS J081554.26+472947.5 & 18.53 & -2.40 & -203.03 & 10.07 & 358.53 & 18.59 & -203.03 & -1.91 \\
SDSS J091753.19+523004.9 & -171.78 & 22.03 & 191.17 & 9.21 & 358.33 & -172.35 & 191.17 & 17.03 \\
SDSS J105002.34+242109.7 & -305.73 & -136.25 & -35.29 & 9.27 & 4.01 & -314.50 & -35.29 & -114.55 \\
SDSS J124304.19-081230.6 & 239.80 & 124.96 & 54.68 & 7.74 & 6.82 & 252.94 & 54.68 & 95.59 \\
SDSS J153346.28+155701.8 & 192.12 & 186.05 & -59.38 & 5.36 & 344.83 & 136.74 & -59.39 & 229.84 \\
\hline
\end{tabular}
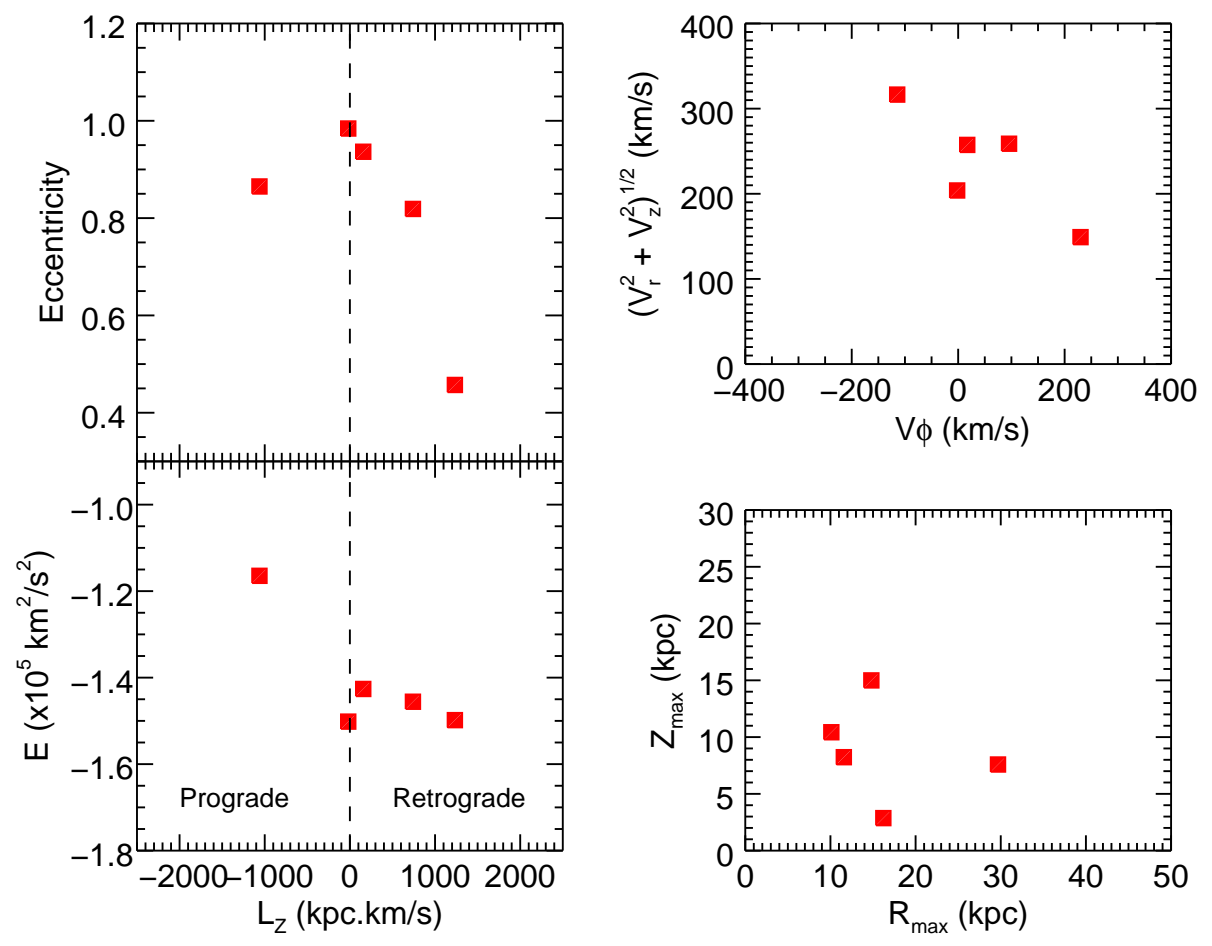

Fig. 3. Orbital parameters for the stars. The eccentricity is computed as $e=\left(R_{\max }-R_{\min }\right) /$ $\left(R_{\max }+R_{\min }\right)$.

positive towards the Galactic anti-centre, $V$ in the direction of the Galactic rotation, and $W$ is perpendicular to the Galactic plane, positive in the northern Galactic hemisphere. We also list the mean specific angular momentum (angular momentum per unit mass) for the stars along their orbits, in units of $\mathrm{kpc} \times \mathrm{km} \mathrm{s}^{-1}$. In Fig. 3 the Toomree diagram and the orbital characteristics of our sample are presented. Four of the stars have large eccentricities and high values of $Z_{\max }$ indicating that they are halo stars. The remaining star with the low $Z_{\max }=2.81 \mathrm{Kpc}$ has an eccentricity of 0.82 and is also likely to have a halo star.

\section{Analysis}

We carried out a classical one-dimension (1D) local thermodynamical equilibrium (LTE) analysis using OSMARCS model atmospheres (Gustafsson et al. 1975, 2003, 2008; Plez et al. 1992; Edvardsson et al. 1993). The abundances used in the model atmospheres were solar-scaled with respect to the
Grevesse \& Sauval (2000) solar abundances, except for the $\alpha$-elements that are enhanced by 0.4 dex. We corrected the resulting abundances by taking into account the difference between the solar abundances of Grevesse \& Sauval (2000), Caffau et al. (2011b), and Lodders et al. (2009). To summarise, the solar abundances adopted for this work are $\log (\mathrm{C} / \mathrm{H})_{\odot}=8.50, \quad \log (\mathrm{Mg} / \mathrm{H})_{\odot}=7.54, \quad \log (\mathrm{Ca} / \mathrm{H})_{\odot}=6.33$, $\log (\mathrm{Fe} / \mathrm{H})_{\odot}=7.52, \log (\mathrm{Sr} / \mathrm{H})_{\odot}=2.92$, and $\log (\mathrm{Ba} / \mathrm{H})_{\odot}=2.17$.

The abundance analysis was performed using the LTE spectral line analysis code turbospectrum (Alvarez \& Plez 1998; Plez 2012), which treats scattering in detail. The carbon abundance was determined by fitting the $\mathrm{CH}$ band near to $430 \mathrm{~nm}$ ( $G$ band). The molecular data that correspond to the $\mathrm{CH}$ band are described in Hill et al. (2002). The abundances were determined by matching a synthetic spectrum centred on each line of interest to the observed spectrum. Table 4 gathers the list of lines used to measure the abundances or evaluate upper limits in our sample of stars. 
Table 4. Absorption lines used to determine the abundances.

\begin{tabular}{lrrr}
\hline \hline Element & Wavelength $(\AA)$ & $\chi_{\text {esc }}$ & $\log g f$ \\
\hline LiI & 6707.761 & 0.00 & -0.009 \\
LiI & 6707.912 & 0.00 & -0.309 \\
CH band & 4315 & & \\
CH band & 4324 & & \\
MgI & 5172.698 & 2.71 & -0.38 \\
MgI & 5183.619 & 2.72 & -0.16 \\
CaI & 4226.740 & 0.00 & +0.24 \\
FeI & 4202.040 & 1.48 & -0.70 \\
FeI & 4260.486 & 2.40 & -0.02 \\
FeI & 4271.164 & 2.45 & -0.35 \\
FeI & 4325.775 & 1.61 & -0.01 \\
FeI & 4383.557 & 1.48 & 0.20 \\
FeI & 4404.761 & 1.56 & -0.14 \\
FeI & 4415.135 & 1.61 & -0.61 \\
FeI & 5269.550 & 0.86 & -1.32 \\
SrII & 4215.520 & 0.00 & -0.17 \\
BaII & 4554.036 & 0.00 & +0.16 \\
\hline
\end{tabular}

Table 5. Estimated errors in the element abundance ratios $[\mathrm{X} / \mathrm{Fe}]$ for the star SDSS J091934.08+524014.0.

\begin{tabular}{lccc}
\hline \hline$[\mathrm{X} / \mathrm{Fe}]$ & $\Delta T_{\text {eff }}=100 \mathrm{~K}$ & $\Delta \log g=0.5 \mathrm{dex}$ & $\Delta v_{t}=0.5 \mathrm{~km} \mathrm{~s}^{-1}$ \\
\hline $\mathrm{C}$ & 0.2 & 0.2 & 0.1 \\
$\mathrm{Mg}$ & 0.1 & 0.15 & 0.15 \\
$\mathrm{Ca}$ & 0.1 & 0.1 & 0.15 \\
$\mathrm{Sr}$ & 0.1 & 0.2 & 0.25 \\
$\mathrm{Ba}$ & 0.1 & 0.2 & 0.3 \\
\hline
\end{tabular}

Notes. The other stars give similar results.

\section{Errors}

Table 5 lists the computed errors in the elemental abundance ratios due to typical uncertainties in the stellar parameters. The errors were estimated varying $T_{\text {eff }}$ by $\pm 100 \mathrm{~K}, \log g$ by $\pm 0.5 \mathrm{dex}$, and $v_{t}$ by $\pm 0.5 \mathrm{dex}$ in the model atmosphere of SDSS J091934.08+524014.0, other stars give similar results. In this star, we were able to measure $\mathrm{Mg}$ and $\mathrm{Ca}$ abundances, and set limits for those of $\mathrm{Li}, \mathrm{Sr}$, and $\mathrm{Ba}$. The main uncertainty comes from the error in the placement of the continuum when the synthetic line profiles are matched to the observed spectra. In particular, residuals from the sky subtraction may lead to a decrease of the $\mathrm{S} / \mathrm{N}$. As the final spectra are built from the combination of several exposures taken at different epochs and therefore different barycentric velocities, the absorption features from the sky residuals are smoothed and degrade the $\mathrm{S} / \mathrm{N}$ of the spectra. This error is of the order of 0.1 to 0.2 depending on the $\mathrm{S} / \mathrm{N}$ of the spectrum and the species under consideration, the largest value being for the neutron capture elements. When several lines are available, the typical line-to-line scatter for a given elements is 0.1 to 0.2 dex.

\section{Results and discussion}

The abundance and upper limit results for the sample of stars of this programme are gathered in Table 6. For lithium and carbon, the $\log (\mathrm{X} / \mathrm{H})$ is given, whereas $[\mathrm{X} / \mathrm{Fe}]$ results are presented for magnesium, calcium, strontium, and barium. For the star SDSS J081554.26+472947.5, the abundance ratios are given assuming $[\mathrm{Fe} / \mathrm{H}]<-4.10 \mathrm{dex}$. This upper limit of the $[\mathrm{Fe} / \mathrm{H}]$ was derived using the strongest FeI line available in our spectrum.

\subsection{Lithium}

Figure 4 shows the abundance of lithium as a function of $[\mathrm{Fe} / \mathrm{H}]$. Our results are represented as red squares. We added the lithium abundances in unevolved stars from the literature (Aoki et al. 2002, 2008; Behara et al. 2010; Bonifacio et al. 2012, 2015; Caffau et al. 2012, 2013, 2016; Carollo et al. 2012; Cohen et al. 2013; Frebel et al. 2007, 2008; González Hernández et al. 2008; Hansen et al. 2014, 2015; Li et al. 2015; Lucatello et al. 2003; Masseron et al. 2012; Matsuno et al. 2017; Norris et al. 1997; Placco et al. 2016; Roederer et al. 2014; Sbordone et al. 2010; Sivarani et al. 2006; Spite et al. 2013).

Normal carbon stars are represented as black circles. Lowand high-carbon-band stars are shown as grey circles. The classification of CEMP stars follows the scheme from Bonifacio et al. (2018) who proposed:

- "Carbon normal" : for $[\mathrm{Fe} / \mathrm{H}] \geq-4.0[\mathrm{C} / \mathrm{Fe}]<1.0$, for $[\mathrm{Fe} / \mathrm{H}]<-4.0 \operatorname{dex} A(\mathrm{C})<5.5$;

- low-carbon-band CEMP stars: stars that do not fulfil the carbon normal criterion and have $A(\mathrm{C}) \leq 7.6$;

- high-carbon-band CEMP stars: stars that do not fulfil the carbon normal criterion and have $A(\mathrm{C})>7.6$.

Different symbols are used for CEMP and non-CEMP to show that the high-carbon-band CEMP stars are preferentially Lidepleted whereas the lithium abundances in the low-carbon-band stars are indistinguishable from those of the carbon-normal stars. These measurements are consistent with the hypothesis suggested by Bonifacio et al. (2018) that the high-carbon-band CEMP stars are the result of mass transfer from an AGB companion, which was also suggested by the works of Koch et al. (2011) and Monaco et al. (2012).

We evaluated the upper limit of the lithium abundance in all the stars of our sample. Four of them reveal a lithium abundance lower than the Spite plateau value (Spite \& Spite 1982; Bonifacio et al. 2007; Sbordone et al. 2010). These stars have metallicities below $[\mathrm{Fe} / \mathrm{H}]=-3.7$ dex, placing them in the region where the "meltdown" of the lithium plateau appears (Sbordone et al. 2010; Aoki et al. 2009; Bonifacio et al. 2007). The star SDSS J081554.26+472947.5, identified as an EMP star by Carbon et al. (2017) and Aguado et al. (2018), has been studied in detail by González Hernández et al. (2020) who found a metallicity of $[\mathrm{Fe} / \mathrm{H}]=-5.49$ dex while we find an upper limit of -4.10 dex. It is interesting to note that by adding more data in this metallicity range, the decrease of the lithium abundance is not constant at a given metallicity. In particular, two stars analysed by Bonifacio et al. (2018) with a metallicity $[\mathrm{Fe} / \mathrm{H}] \simeq-4.00 \mathrm{dex}$ show a lithium abundance at the level of the Spite plateau. From Fig. 4, it seems that the Spite plateau appears as an upper limit for the lithium abundance in metal-poor unevolved stars. As the metallicity decreases below $[\mathrm{Fe} / \mathrm{H}]=-3.0$ dex, the dispersion of the lithium abundance at a given metallicity seems to increase with decreasing metallicity.

\subsection{Carbon}

Figure 5 shows the abundance of carbon as a function of $[\mathrm{Fe} / \mathrm{H}]$. The results are represented as red squares with downwardpointing arrows, which indicate that the abundances are upper limits. Open red symbols represent the stars for which we find low [n-capture/Fe] upper limit abundances. Other stars from the 
Table 6. Lithium and carbon abundances.

\begin{tabular}{lrrrrrrr}
\hline \hline Object & {$[\mathrm{Fe} / \mathrm{H}]$} & $A(\mathrm{Li})$ & $A(\mathrm{C})$ & {$[\mathrm{Mg} / \mathrm{Fe}]$} & {$[\mathrm{Ca} / \mathrm{Fe}]$} & {$[\mathrm{Sr} / \mathrm{Fe}]$} & {$[\mathrm{Ba} / \mathrm{Fe}]$} \\
\hline SDSS JJ091753.19+523004.9 & -3.70 & $\leq 1.8$ & $\leq 6.7$ & 0.46 & 0.27 & $\leq-0.42$ & $\leq 0.23$ \\
SDSS J105002.34+242109.7 & -4.25 & $\leq 2.0$ & $\leq 6.8$ & 0.41 & $\leq-0.08$ & $\leq 0.53$ & $\leq 1.18$ \\
SDSS J124304.19-081230.6 & -4.05 & $\leq 1.7$ & $\leq 7.0$ & 0.21 & -0.28 & $\leq-0.67$ & $\leq 0.08$ \\
SDSS J153346.28+155701.8 & -3.50 & $\leq 2.2$ & $\leq 7.0$ & 0.26 & 0.07 & $\leq-0.22$ & $\leq-0.17$ \\
\hline Object & {$[\mathrm{Fe} / \mathrm{H}]$} & $A(\mathrm{Li})$ & $A(\mathrm{C})$ & {$[\mathrm{Mg} / \mathrm{H}]$} & {$[\mathrm{Ca} / \mathrm{H}]$} & {$[\mathrm{Sr} / \mathrm{H}]$} & {$[\mathrm{Ba} / \mathrm{H}]$} \\
\hline SDSS J081554.26+472947.5 & $\leq-4.10$ & $\leq 2.0$ & $\leq 7.3$ & -3.67 & $\leq-4.13$ & $\leq-3.42$ & $\leq-2.77$ \\
\hline
\end{tabular}

Notes. $\alpha$ and neutron-capture element abundance ratios are shown in the first four rows. The $[\mathrm{Fe} / \mathrm{H}]$ abundance for SDSS J081554.26+472947.5 is an upper limit. Therefore, the abundances are given as $A(\mathrm{X})$ for $\mathrm{Li}$ and $\mathrm{C}$ and $[\mathrm{X} / \mathrm{H}]$ for $\mathrm{Fe}, \mathrm{Mg}, \mathrm{Ca}, \mathrm{Sr}$ and $\mathrm{Ba}$.

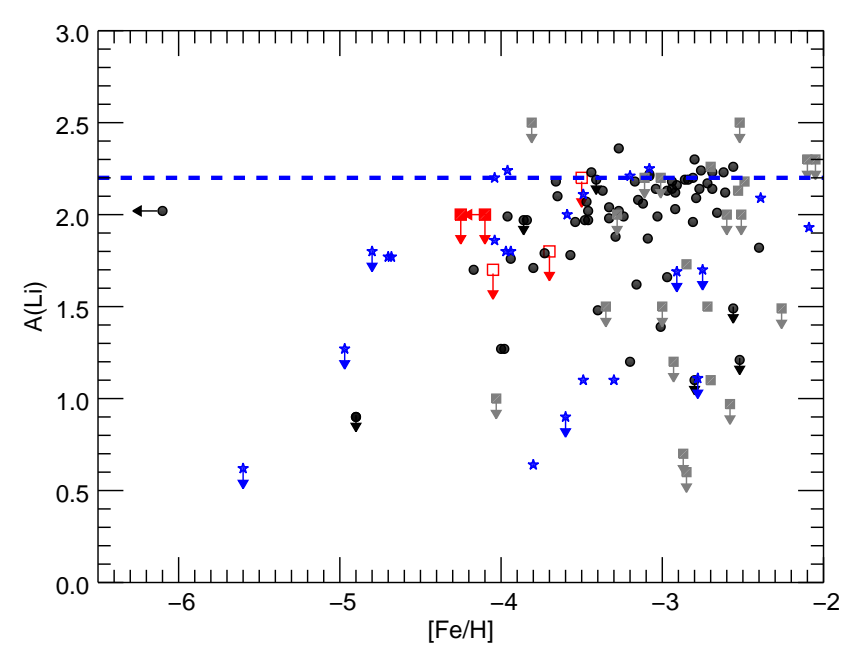

Fig. 4. Lithium abundance in unevolved EMP stars. Filled circles refer to carbon-normal stars. CEMP stars of the low- and high-carbon bands are shown as blue stars and grey rectangles, respectively. Upper limits of the programme stars are shown in red. Open red symbols represent the stars for which we find low [n-capture/Fe] upper limit abundances. The blue dashed line represents the Spite Plateau as determined by Sbordone et al. (2010). Details about literature data (black and grey symbols) can be found in Bonifacio et al. (2018). The star SDSSJ002314.00+030758.07 with $[\mathrm{Fe} / \mathrm{H}]<-6.10$ dex from Aguado et al. (2019) has been added.

literature are shown for reference (Aoki et al. 2008; Behara et al. 2010; Cohen et al. 2013; Frebel et al. 2005, 2006; Li et al. 2015; Masseron et al. 2010; Sivarani et al. 2006; Plez \& Cohen 2005; Plez et al. 2005; Thompson et al. 2008; Yong et al. 2013). The upper limits of the carbon abundance we find in our five stars are compatible with them being moderately enhanced in $\mathrm{C}$ or $\mathrm{C}$ normal. As our measurements are upper limits, the stars could be CEMP stars belonging to the low carbon band or could be carbon-normal stars.

\section{3. $\alpha$ and neutron-capture elements}

Figure 6 shows the abundance ratios of $[\mathrm{Mg} / \mathrm{Fe}]$ and $[\mathrm{Ca} / \mathrm{Fe}]$ as a function of $[\mathrm{Fe} / \mathrm{H}]$ for our sample of stars using the solar abundances from Lodders et al. (2009). Literature data from Roederer et al. (2014) are also shown, which contain results from a rather large sample of evolved and main sequence stars. We were able to measure magnesium in the five stars. Among them, SDSS J081554.26+472947.5 seems to have a rather low $[\mathrm{Mg} / \mathrm{Fe}]$ abundance ratio and a subsolar $[\mathrm{Ca} / \mathrm{Fe}]$ upper limit abundance. This is due to our high upper limit of $[\mathrm{Fe} / \mathrm{H}]$. In

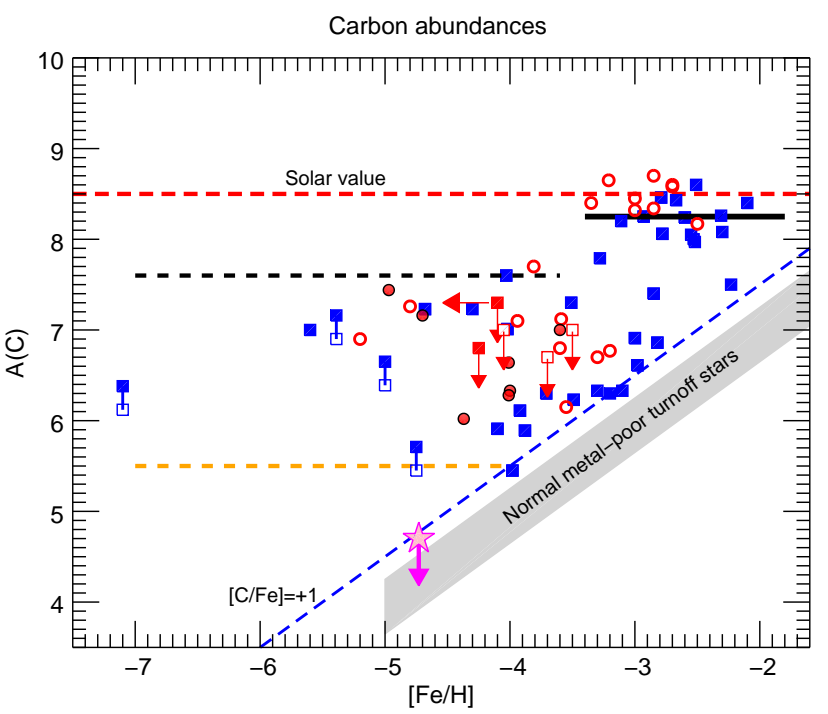

Fig. 5. Carbon abundances $A(C)$ of CEMP stars as a function of $[\mathrm{Fe} / \mathrm{H}]$. Large filled red squares represent our results. Downward-pointing arrows indicate that the abundances are given as upper limits. Open red symbols represent the stars for which we find low [n-capture/Fe] upper limit abundances. Open and filled red circles are data published by our group. Other stars from the literature are represented as blue squares (see main text for details). Open blue squares are carbon abundances derived using 3D corrections from Gallagher et al. (2016). Black circles filled in red are stars from Bonifacio et al. (2018). The pink symbol represents SDSS J102915+17292, the normal-carbon, ultra metal-poor star discovered by Caffau et al. (2011a). The other symbols are literature data. The black and yellow dashed lines delimit the low-carbon band. Details can be found in Bonifacio et al. (2018).

fact, by adopting the very low $[\mathrm{Fe} / \mathrm{H}]$ determined by González Hernández et al. (2020), we obtain $[\mathrm{Mg} / \mathrm{Fe}]=1.62$ dex in excellent agreement with the $[\mathrm{Mg} / \mathrm{Fe}]=1.66 \mathrm{dex}$ abundance that these latter authors obtained. The stellar parameters we adopted for SDSS J081554.26+472947.5 are close to those used by González Hernández et al. (2020) with a difference of $15 \mathrm{~K}$ on the temperature, a difference of 0.6 dex in $\log g$ that has no strong effect (typically 0.1 to $0.15 \mathrm{dex}$ on the neutral $\mathrm{Ca}$ and $\mathrm{Mg}$ ) on the determination of the abundance of neutral species, and the same micro-turbulent velocity. We found three stars with subsolar values for calcium, confirming the existence of stars with low $[\alpha / \mathrm{Fe}]$ ratios as already suggested by Bonifacio et al. (2018). However, we reiterate the fact that in very metal-poor stars, under the LTE hypothesis, the resonance line that we used to determine the abundance of calcium leads to an underestimation of 

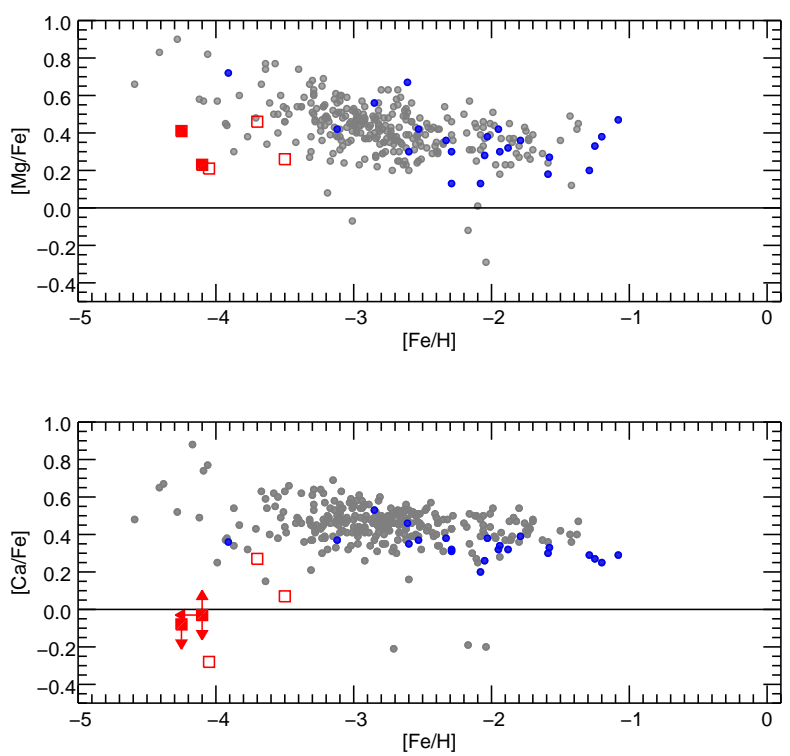

Fig. 6. $[\mathrm{Mg} / \mathrm{Fe}]$ and $[\mathrm{Ca} / \mathrm{Fe}]$ vs. $[\mathrm{Fe} / \mathrm{H}]$. Red squares are results from the present study. Open red squares represent the stars for which we find low [n-capture/Fe] upper limit abundances. Grey circles show evolved stars from Roederer et al. (2014). Blue circles show main sequence stars from Roederer et al. (2014).

the calcium abundance (Spite et al. 2012, and references therein). For turnoff stars, the amplitude of the effect is rather small. Spite et al. (2012) computed a correction of the order of +0.1 dex in a turnoff star with $[\mathrm{Fe} / \mathrm{H}]=-3.2$ dex. Figure 7 shows the $[\mathrm{Mg} / \mathrm{Ca}]$ ratio as a function $[\mathrm{Fe} / \mathrm{H}]$ (upper panel) and as a function of $[\mathrm{Mg} / \mathrm{H}]$ (lower panel). We have added the literature data from Roederer et al. (2014) for comparison. The $[\mathrm{Mg} / \mathrm{Ca}]$ ratio found in our sample of stars ranges from +0.2 to $+0.5 \mathrm{dex}$. This ratio is slightly higher than the abundance ratio found in the $[\mathrm{Fe} / \mathrm{H}]$ range of -2 to -3 dex. At lower metallicities, the results from Roederer et al. (2014) show that the spread in the $[\mathrm{Mg} / \mathrm{Ca}]$ ratio increases as $[\mathrm{Fe} / \mathrm{H}]$ decreases with values ranging from negative abundance ratios to highly enhanced $[\mathrm{Mg} / \mathrm{Ca}]$ ratios. From our measurements, the $[\mathrm{Mg} / \mathrm{Ca}]$ ratio seems to increase as $[\mathrm{Fe} / \mathrm{H}]$ decreases. The result for SDSS J081554.26+472947.5 found by González Hernández et al. (2020) seems to corroborate this point. The increase of the $[\mathrm{Mg} / \mathrm{Ca}]$ ratio is also visible when the ratio is plotted as a function of $[\mathrm{Mg} / \mathrm{H}]$. However, a high value of $[\mathrm{Mg} / \mathrm{Ca}]$ at very low $[\mathrm{Mg} / \mathrm{H}]$ is not found in all the stars. In particular, SDSS J102915+17292, the normal carbon ultra metalpoor star discovered by Caffau et al. (2011a), has a solar [Mg/Ca] ratio. Further observations at metallicities below $[\mathrm{Fe} / \mathrm{H}]=-4 \mathrm{dex}$ are necessary to conclude whether there is indeed an increase of the ratio $[\mathrm{Mg} / \mathrm{Ca}]$ with decreasing metallicity or an increase of the spread of the $[\mathrm{Mg} / \mathrm{Ca}]$ and a possible link to the $\mathrm{C}$ abundance found in the star.

Figure 8 shows the abundance ratios and upper limits of $[\mathrm{Sr} / \mathrm{Fe}]$ and $[\mathrm{Ba} / \mathrm{Fe}]$ as a function of $[\mathrm{Fe} / \mathrm{H}]$. We have also added the results from Roederer et al. (2014), which encompass data from a rather large sample of evolved and main sequence stars analysed in a homogeneous way. Although we only determine upper limits, it is interesting to note that our stars can be divided into two groups, one with a high [n-capture/Fe] ratio, typically $[\mathrm{Sr} / \mathrm{Fe}] \simeq+0.5$ to +0.7 dex and $[\mathrm{Ba} / \mathrm{Fe}]$ around $+1.2 \mathrm{dex}$, and $\mathrm{a}$ second with solar $[\mathrm{Sr} / \mathrm{Fe}] \simeq-0.4$ to $-0.7 \mathrm{dex}$ and solar $[\mathrm{Ba} / \mathrm{Fe}]$ ratios. The low [n-capture/Fe] found in the group of three stars is generally found in stars in the metallicity range around -2.5 dex.
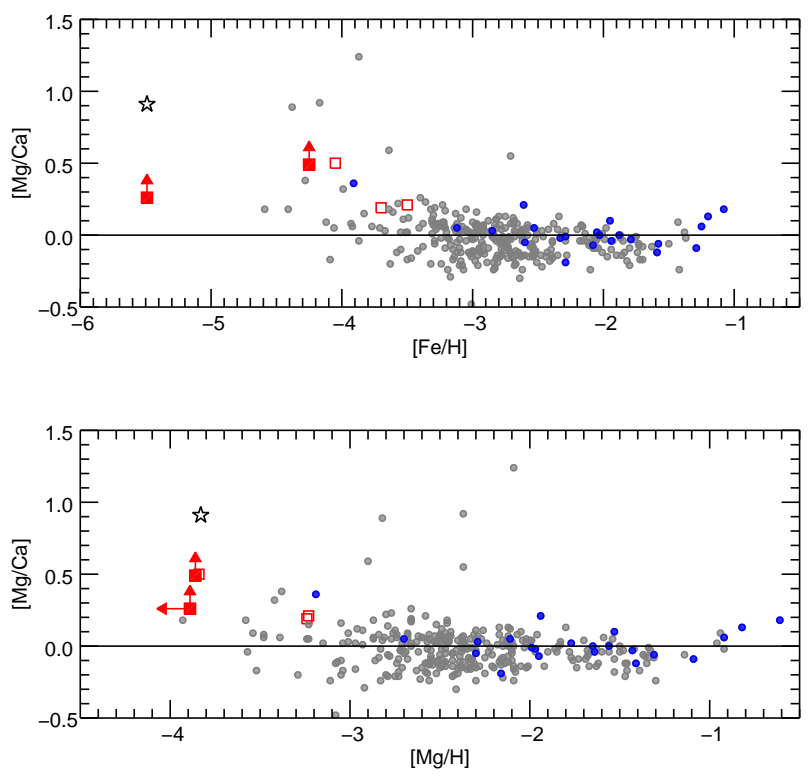

Fig. 7. $[\mathrm{Mg} / \mathrm{Ca}]$ vs. $[\mathrm{Fe} / \mathrm{H}]$ and $[\mathrm{Mg} / \mathrm{Ca}]]$ vs. $[\mathrm{Mg} / \mathrm{H}]$. Red squares show results from the present study. Open red squares represent the stars for which we find low [n-capture/Fe] upper-limit abundances. Grey circles indicate evolved stars from Roederer et al. (2014), blue circles show main sequence stars from Roederer et al. (2014), and the black open symbol represents SDSS J081554.26+472947.5 (González Hernández et al. 2020).
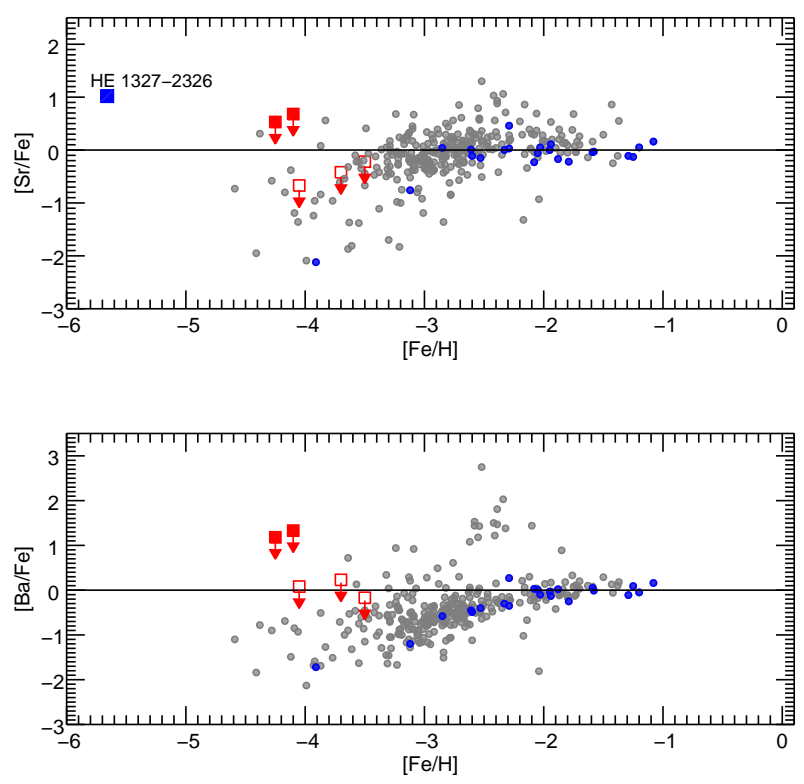

Fig. 8. $\mathrm{Sr} / \mathrm{Fe}]$ and $[\mathrm{Ba} / \mathrm{Fe}]$ vs. $[\mathrm{Fe} / \mathrm{H}]$. Red squares indicate results from the present study. Open red squares represent the stars for which we find low [n-capture/Fe] upper limit abundances. Grey circles show evolved stars from Roederer et al. (2014), blue circles indicate main sequence stars from Roederer et al. (2014), and the blue rectangle represents HE1327-2326, a star with an exceptionally high [Sr/Fe] ratio (Frebel et al. 2008; Aoki et al. 2006).

Two stars display a very high [n-capture/Fe] similar to the exceptionally high $[\mathrm{Sr} / \mathrm{Fe}]$ ratio found in the HE1327-2326 (Frebel et al. 2008; Aoki et al. 2006). New observations with better S/N ratios could be used to firmly determine the abundance ratios found in these stars. 


\section{Conclusions}

In this article, we reported the chemical analysis of five extremely metal-poor candidates observed with the high dispersion spectrograph (HDS) at the Subaru telescope. We discover a new UMP star, SDSS J105002.34+242109.7, with $[\mathrm{Fe} / \mathrm{H}]=$ -4.25 dex. We were able to determine the abundances of some elements $(\mathrm{C}, \mathrm{Mg}, \mathrm{Ca}, \mathrm{Sr}$ and $\mathrm{Ba})$ in the majority of these stars. The five stars of the sample show abundance ratios which are typical of metal-poor stars in the metallicity range -4.25 dex $\leq$ $[\mathrm{Fe} / \mathrm{H}] \leq-3.5$ dex. These results show that the method developed by Ludwig et al. (2008) to estimate the metallicity of unevolved stars from low-resolution spectra is very efficient. We were able to measure an upper limit for the lithium abundance in the five stars of our sample. The four most-metal-poor stars, with a metallicity ranging from -3 to -4.25 dex, show lithium abundances below the Spite plateau. Some stars of our sample show a low $[\alpha / \mathrm{Fe}]$ content, a characteristic already found in previous studies (Bonifacio et al. 2018, an reference therein). For the star SDSS J081554.26+472947.5 we obtain a high $[\mathrm{Mg} / \mathrm{Fe}]$ of +1.62 dex if we adopt the metallicity measured by González Hernández et al. (2020).

Acknowledgements. This work was supported by JSPS and CNRS under the Japan-France Research Cooperative Program (CNRS PRC No 1363), the JSPS Grants-in-Aid for Scientific Research (26400232, 26400237), and the RIKEN iTHEMS Project. P.F. acknowledges support by the Conseil Scientifique de l'Observatoire de Paris (AFE programme). We gratefully acknowledge support from the French National Research Agency (ANR) funded project "Pristine" (ANR-18-CE31-0017)

\section{References}

Adams, W. S., Joy, A. H., Humason, M. L., et al. 1935, ApJ, 81, 187

Aguado, D., Allende Prieto, C., González Hernandez, J., \& Rebolo, R. 2018, ApJ, 854, 34

Aguado, D. S., Hernández, J. I. G., Prieto, C. A., et al. 2019, ApJ, 874, L21

Alvarez, R., \& Plez, B. 1998, A\&A, 330, 1109

Aoki, W., Christlieb, N., Norris, J. E., et al. 2006, ApJ, 639, 897

Aoki, W., Beers, T. C., Sivarani, T., et al. 2008, ApJ, 678, 1351

Aoki, W., Barklem, P., Beers, T. C., et al. 2009, ApJ, 698, 1803

Aoki, W., Ryan, S. G., \& Norris, J. E. 2020, ApJ, 580, 1149

Arenou, F., Luri, X., Babusiaux, C., et al. 2018, A\&A, 616, A17

Bailer-Jones, C. A. L., Rybizki, J., Fouesneau, M., Mantelet, G., \& Andrae, R. 2018, AJ, 156, 58

Barklem, P. S., Christlieb, N., Beers, T. C., et al. 2005, A\&A, 439, 129

Baschek, B. 1963, Z. Astrophys., 56, 207

Behara, N. T., Bonifacio, P., Ludwig, H.-G., et al. 2010, A\&A, 513, A72

Beers, T., \& Christlieb, N. 2005, ARA\&A, 43, 531

Beers, T., Preston, G. W., \& Schectman, S. A., 1985, AJ, 90, 2089

Bonifacio, P., \& Monai Beers, T. C. 2000, AJ, 120, 2065

Bonifacio, P., Molaro, P., Sivarani, T., et al. 2007, A\&A, 462, 85

Bonifacio, P., Spite, M., \& Cayrel, R. 2009, A\&A, 501, 519

Bonifacio, P., Sbordone, L., Caffau, E., et al. 2012, A\&A, 542, A87

Bonifacio, P., Caffau, E., Spite, M., et al. 2015, A\&A, 579, A28

Bonifacio, P., Caffau, E., Spite, M., et al. 2018, A\&A, 612, A65

Bressan, A., Marigo, P., Girardi, L., et al. 2012, MNRAS, 427, 127

Caffau, E., Bonifacio, P., François, P., et al. 2011a, Nature, 477, 67

Caffau, E., Ludwig, H.-G., Steffen, M., Freytag, B., \& Bonifacio, P. 2011b, Sol. Phys., 268, 255

Caffau, E., Bonifacio, P., François, et al. 2012, A\&A, 542, A51

Caffau, E., Bonifacio, P., François, et al. 2013, A\&A, 560, A15

Caffau, E., Sbordone, L., Bonifacio, P., et al. 2014, Mem. Soc. Astron. It., 85, 222

Caffau, E., Bonifacio, P., Spite, M., et al. 2016, A\&A, 595, A6

Carollo, D., Beers, T. C., \& Bovy, J. 2012, ApJ, 744, 195

Carbon, D., Henze, C., \& Nelson, B. 2017, ApJS, 228, 19

Cayrel, G., \& Fringant, A.-M. 1964, C. R. Acad. Sc. Paris, 258, 3195

Cayrel, R., Depagne, E., Spite, M., et al. 2004, A\&A, 416, 1117
Chamberlain, J. W., \& Aller, L. H. 1951, ApJ, 114, 52

Christlieb, N., Schorck, T., Frebel, A., et al 2008, A\&A, 484, 721

Cohen, J. G., Christlieb, N., Thompson, I., et al. 2013, ApJ, 778, 56

Dehnen, W., \& Binney, J. 1998, MNRAS, 294, 429

Edvardsson, B., Andersen, J., Gustafsson, B., et al. 1993, A\&A, 275, 101

Francois, P. , Caffau, E., Wanajo, S., et al. 2018, A\&A, 619, A10

Frebel, A., \& Norris, J. E. 2015, ARA\&A, 53, 631

Frebel, A., Aoki, W., Christlieb, N., et al. 2005, Nature, 434, 871

Frebel, A., Christlieb, N., Norris, J. E., et al. 2006, ApJ, 652, 1585

Frebel, A., Norris, J. E., Aoki, W., et al. 2007, ApJ, 658, 534

Frebel, A., Collet, R., Eriksson, K., Christlieb, N., \& Aoki, W. 2008, ApJ, 684, 588

Gaia Collaboration (Brown, A. G. A., et al.) 2018, A\&A, 616, A1

Greenstein, J. L., Hack, M., \& Struve, O. 1957, ApJ, 126, 281

Gallagher, A. J., Caffau, E., Bonifacio, P., et al. 2016, A\&A, 593, A48

González Hernández, J. I., Bonifacio, P., Ludwig, H.-G., et al. 2008, A\&A, 480, 233

González Hernández, J. I., Aguado, D. S., Prieto, C. A., et al. 2020, ApJ, 889, L13

Grevesse, N., \& Sauval, A. J. 2000, Origin of Elements in the Solar System, ed. O. Manuel (BostonL Springer), 261

Gustafsson, B., Bell, R. A., Eriksson, K., \& Nordlund, A. 1975, A\&A, 42, 407

Gustafsson, B., Edvardsson, B., Eriksson, K., et al. 2003, ASP Conf. Ser., 288, 331

Gustafsson, B., Edvardsson, B., Eriksson, K., et al. 2008, A\&A, 486, 951

Hansen, T., Hansen, C. J., Christlieb, N., et al. 2014, ApJ, 787, 162

Hansen, T., Hansen, C. J., Christlieb, N., et al. 2016, ApJ, 807, 173

Helmi, A., Ivezić, Ž, Prada, F., et al. 2003, ApJ, 586, 195

Hill, V., Plez, B., Cayrel, R., et al. 2002, A\&A, 387, 560

Keller, S. C., Schmidt, B. P., Bessell, M. S., et al. 2007, Publ. Astron. Soc. Aust., 24,1

Keller, S. C., Bessell, M. S., Frebel, A., et al. 2014, Nature, 506, 463

Kelson, D. 2003, PASP, 115, 688

Koch, A., Lind, K., \& Rich, R. M. 2011, ApJ, 738, L29

Li, H.-N., Zhao, G., Christlieb, N., et al. 2015, ApJ, 798, 110

Lodders, K., Plame, H., \& Gail, H.-P. 2009, Landolt-Börnstein - Group VI Astronomy and Astrophysics Numerical Data and Functional Relationships in Science and Technology Volume 4B: Solar System, ed. J. E. Trümper, 4.4., 44 Lucatello, S., Gratton, R., Cohen, J. G. 2003, AJ, 125, 875

Ludwig, H. G., Bonifacio, P., Caffau, E., et al. 2008, Phys. Scr., 133

Masseron, T., Johnson, J. A., Plez, B., et al. 2010, A\&A, 509, A93

Masseron, T., Johnson, J., \& Lucatello, S. 2012, ApJ, 751, 14

Monaco, L., Villanova, S., \& Bonifacio, P. 2016, A\&A, 539, A157

Matsuno, T., Aoki, W., Beers, T. C., Lee, Y. S., \& Honda, S. 2017, AJ, 154, 52

Majewski, S. R., \& APOGEE Team, 2016, Astron. Nachr., 337, 863

Meynet, G., Hirschi, R., Ekstrom, S., et al. 2010, A\&A, 521, A30

Noguchi, K., Aoki, W., Kawanomoto, S., et al. 2002, PASJ, 54, 855

Nordlander, T., Bessell, M. S., Da Costa, G. S., et al. 2019, MNRAS, 488, L109

Norris, J., Peterson, R., Beers, T., 1993, ApJ, 415, 797

Norris, J., Ryan, S. G., Beers, T. C., \& Deliyannis, C. P. 1997, ApJ, 485, 370

Placco, V. M., Beers, T. C., Reggiani, H., \& Meléndez, J. 2016, ApJ, 829, L24

Plez, B. 2012, Astrophysics Source Code Library [record ascl:1205.004]

Plez, B., \& Cohen, J. G. 2005, A\&A, 434, 1117

Plez, B., Brett, J. M., \& Nordlund, A. 1992, A\&A, 256, 551

Plez, B., Cohen, J. G., \& Meléndez, J. 2005, IAU Symp., 228, 267

Roederer, I. U., Preston, G. W., \& Thompson, I. B., et al. 2014, AJ, 147, 136

Roman, N. 1955, ApJS, 2, 195

Sbordone, L., Bonifacio, P., Caffau, E., et al. 2010, A\&A, 522, A26

Schlafly, E. F., \& Finkbeiner, D. P. 2011, ApJ, 737, 103

Schlegel, D. J., Finkbeiner, D. P., Davis, M. 1998, ApJ, 500, 525

Sivarani, T., Beers, T. C., Bonifacio, P., et al. 2006, A\&A, 459, 125

Spite, F., \& Spite, M. 1982, A\&A, 115, 357

Spite, M., Andrievsky, S., Spite, F., et al. 2012, A\&A, 541, A143

Spite, M., Caffau, E., Bonifacio, P., et al. 2013, A\&A, 552, A107

Starkenburg, E., Martin, N., Youakim, K., et al. 2017, MNRAS, 471, 2587

Steimetz, M., Zwitter, T., Siebert, A., et al. 2006, AJ, 132, 4

Thompson, I. B., Ivans, I. I., Bisterzo, S., et al. 2008, ApJ, 677, 556

Wallerstein, G. 1962, ApJS, 6, 407

Wallerstein, G., \& Helfer, H. L. 1959, ApJ, 129, 720

Wallerstein, G., Greenstein, J. L., Parker, R., et al. 1963, ApJ, 137, 280

Wolf, C., Onken, C. A., Luvaul, L. C., et al. 2018, Publ. Astron. Soc. Aust., 35, $\mathrm{e} 010$

Yong, D., Norris, J., Bessel, M., et al. 2013, ApJ , 762, 27 